\title{
Footstep Parameterized Motion Blending using Barycentric Coordinates
}

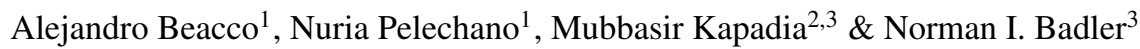 \\ ${ }^{1}$ Universitat Politècnica de Catalunya \\ ${ }^{2}$ Rutgers University \\ ${ }^{3}$ University of Pennsylvania
}

\begin{abstract}
This paper presents a real-time animation system for fully-embodied virtual humans that satisfies accurate foot placement constraints for different human walking and running styles. Our method offers a fine balance between motion fidelity and character control, and can efficiently animate over sixty agents in real time (25 FPS) and over a hundred characters at 13 FPS. Given a point cloud of reachable support foot configurations extracted from the set of available animation clips, we compute the Delaunay triangulation. At runtime, the triangulation is queried to obtain the simplex containing the next footstep, which is used to compute the barycentric blending weights of the animation clips. Our method synthesizes animations to accurately follow footsteps, and a simple IK solver adjusts small offsets, foot orientation, and handles uneven terrain. To incorporate root velocity fidelity, the method is further extended to include the parametric space of root movement and combine it with footstep based interpolation. The presented method is evaluated on a variety of test cases and error measurements are calculated to offer a quantitative analysis of the results achieved.
\end{abstract}

Keywords:

Character animation, Crowd simulation, Footsteps controller

\section{Introduction}

2 Crowd simulation research has matured in recent years with з important applications in training, building design, psycholog4 ical studies, and video-games. All these applications benefit 5 from having fully-embodied virtual human characters animated 6 in real-time while accurately satisfying control objectives with7 out any noticeable artifacts.

8 Algorithms that generate center of mass (COM) trajecto9 ries $[1,2,3,4]$ lead to ambiguities when trying to superimpose 10 a fully articulated virtual human to follow them, thus produc${ }_{11}$ ing foot-sliding artifacts when no suitable animation is found, 12 or when the root orientation and the displacement vector of the 13 animation do not match. Different animations can be blended ${ }_{14}$ by tweaking some of the upper body joints [5] to minimize ar15 tifacts, at the expense of constant updates to account for the de${ }_{16}$ coupling between the crowd simulation and the animation sys17 tem.

18 Footstep-based control systems [6, 7] output a list of space19 time foot-plants to define a fine-grained trajectory with fewer 20 ambiguities that can solve more complex scenarios (e.g., com${ }_{21}$ plex manipulation tasks requiring careful control of the lower 22 body, or collaborative tasks, such as careful sidestepping to 23 make way for another agent in a narrow corridor). To realis24 tically represent such simulations, we need a method to synthe25 size animations that accurately follow the output trajectory, i.e., ${ }_{26}$ accurate placement of feet with space-time constraints. This ${ }_{27}$ problem is traditionally known as the stepping stone problem.
28 Moreover, the output trajectory can be modified by external per29 turbations such as uneven terrain.

We present an online animation synthesis technique for fully ${ }_{31}$ embodied virtual humans that satisfies foot placement constraints ${ }_{32}$ for a large variety of locomotion speeds and styles (see Fig. ${ }_{33} 1$ ). Given a database of motion clips, we precompute multiple ${ }_{34}$ parametric spaces based on the motion of the root and the feet. ${ }_{35} \mathrm{~A}$ root parametric space is used to compute a weight for each ${ }_{36}$ available animation based on root velocity. Two foot paramet${ }_{37}$ ric spaces are based on a Delaunay triangulation of the graph of 38 possible foot landing positions. For each foot parametric space, ${ }_{39}$ blending weights are calculated as the barycentric coordinates ${ }_{40}$ of the next footstep position for the triangle in the graph that ${ }_{41}$ contains it. These weights are used for synthesizing animations 42 that accurately follow the footstep trajectory while respecting 43 the singularities of the different walking styles captured.

$44 \quad$ Blending weights calculated as barycentric coordinates are 45 used to reach the desired foot landing by interpolating between ${ }_{46}$ several proximal animations, and IK is used to adjust the final ${ }_{47}$ position of the support foot to correct for minor offsets, foot 48 step orientation and the angle of the underlying floor.

49 Since foot parametric space only considers final landing po50 sitions of the feet without taking into account root velocity, this 51 may lead to the selection of animations that satisfy position 52 constraints but introduce discontinuities in root velocity. To in${ }_{53}$ corporate root velocity fidelity we present a method that can 54 integrate both foot positioning and root velocity fidelity. Our 55 method also allows the system to recover nicely when the input 

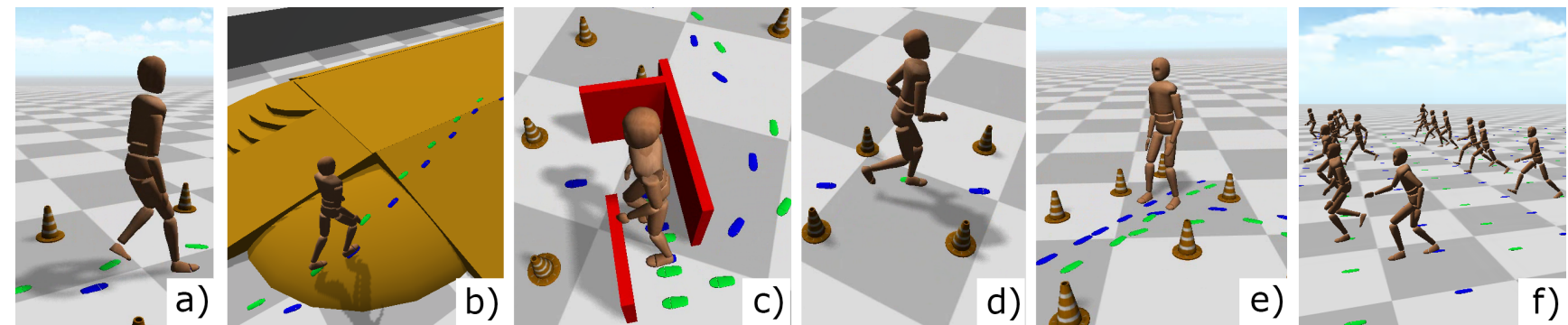

Figure 1: An autonomous virtual human navigating a challenging obstacle course (a), walking over a slope (b), exercising careful foot placement constraints including side-stepping (c), speed variations (d), and stepping back (e). The system can handle multiple agents in real time (f).

${ }_{56}$ foot trajectory contains steps that are not possible to perform 57 with the given set on animations (for example, due to extreme ${ }_{58}$ distance between steps).

59 The presented method is evaluated on a variety of test cases 60 and error measurements are calculated to offer a quantitative ${ }_{61}$ analysis of the results achieved. Our framework can efficiently 62 animate over sixty agents in real time (25 FPS) and over a hun${ }_{63}$ dred characters at 13 FPS, without compromising motion fi${ }_{64}$ delity or character control, and can be easily integrated into ex65 isting crowd simulation packages. We also provide the user 66 with control over the trade-off between footstep accuracy and 67 root velocity.

\section{2. Related Work}

69 Locomotion synthesis can be tackled from different points 70 of view depending on how the character is being controlled. 71 If a user controls the character with a 3rd person controller, it 72 is common to work on a root velocity basis, because the user 73 wants to move the character around in an agile way. In such 74 cases, like video-games, real-time response is critical and arti75 facts such as foot skating can be ignored. Optimization based 76 approaches [8] are able to synthesize animations that conform 77 to velocity and orientation constraints. However, they need a 78 very large database and their computational time does not al79 low many characters in real-time. Semi-procedural animation 80 systems [9] work with a small set of animations and use inverse ${ }_{81}$ kinematics only over the legs to ensure ground contact and to 82 adapt the feet to possible slopes of the terrain, but they are un83 able to follow footstep trajectories.

Animation systems for autonomous agents must be com${ }_{85}$ putationally efficient to animate a multitude of characters in ${ }_{86}$ real-time, and need to follow different control trajectories, de${ }_{87}$ pending on the controller used. Controllers that account for an88 imation constraints while computing control decisions such as 89 motion graphs $[10,11,12,13]$ or precomputed search trees [14] 9o can simply playback the animation sequence. These approaches ${ }_{91}$ try to reach the goal by connecting series of motion[15], which ${ }_{92}$ sometimes limits the movements of the agents. The main issues 93 with motion graphs are that they require a very large amount 94 of animation clips (over 400) and have a high computational ${ }_{95}$ cost which makes them not suitable for large groups of agents ${ }_{96}$ in real-time. Precomputed search trees can handle groups, but
${ }_{97}$ work with a few animation clips and are unable to synthesize 98 new animations.

99 Approaches that ignore animation constraints produce cen100 ter of mass trajectories for the animation system to follow. Dif${ }_{101}$ ferent models include social forces [2], rule-based approaches [1], 102 flow tiles [16], roadmaps [17], continuum dynamics [3], and 103 force models parametrized by psychological and geometrical 104 rules [4]. These techniques can easily simulate hundreds and 105 thousands of characters in real-time, but do not account for 106 locomotion constraints, thus producing artifacts such as foot${ }_{107}$ sliding which require correction and simulation updates [5].

108 Considering the root velocity as the input parameter for 109 character control, numerous approaches can synthesize smooth, 110 versatile and more plausible locomotion animations [18, 9]. Some 111 approaches have also used the idea of selecting animations from 112 a Delaunay triangulation of all the available animation clips ${ }_{113}[19,20]$. But all of these approaches are restricted to the root ${ }_{114}$ for performing character control.

There has been a recent surge in approaches that produce ${ }_{116}$ footstep trajectories for character control. They can be phys117 ically based but generated off-line [21], be generated online 118 from an input path computed by a path planner [6], or use sim${ }_{119}$ plified control dynamics to produce bio-mechanically plausi120 ble footstep trajectories for crowds [7]. These approaches of${ }_{121}$ ten show their animation results off-line using tools such as 3D 122 Max [22].

${ }_{123}$ Footstep-driven animation systems [23] produce unnatural 124 results using procedural methods. The work in [24] uses a sta125 tistical dynamic model learned from motion capture data in ad${ }_{126}$ dition to user-defined space-time constraints (such as footsteps) ${ }_{127}$ to solve a trajectory optimization problem. In [25] random 128 samples of footsteps make a roadmap going from one point 129 to another which is used to find a minimum-cost sequence of 130 motions matching it and then retarget to the exact foot place131 ments. The work in $[26,27]$ performs a global optimization 132 over an extracted center of mass trajectory to maximize the ${ }_{133}$ physical plausibility and perceived comfort of the motion, in 134 order to satisfy the footprint constraints. Recent solutions [6, ${ }_{135} 28,29$ ] adopt a greedy nearest-neighbor approach over larger 136 motion databases. To ensure spatial constraints, the character ${ }_{137}$ is properly aligned with the footsteps and reinforced with in138 verse kinematics, while temporal constraints are satisfied us139 ing time warping. These techniques achieve highly accurate re${ }_{140}$ sults in terms of foot positioning, but their computational cost 
${ }_{141}$ makes them unsuitable for real-time animation of large groups 142 of agents.

${ }_{143}$ Comparison to Prior Work. Our method produces visually ${ }_{144}$ appealing results with foot placement constraints, using only a 145 handful of motion clips, and can seamlessly follow footstep${ }_{146}$ based control trajectories while preserving the global appear147 ance of the motion. Compared to [9], we exploit the combi148 nation of multiple parameter spaces for footstep-precision con149 trol. This reduces the dimensionality of the problem, compared 150 to [29]. Unlike previous work in the literature, our method can ${ }_{151}$ synthesize animations for a large number of characters in real 152 time, following footstep trajectories for different walking styles 153 and even running motions with a small flying phase.

\section{3. Framework Overview}

155 Animating characters in real time animations has different 156 requirements depending on the application. In many applica157 tions, the user only wants to control the direction of movement 158 and speed of the root, but there are other situations where a 159 finer control of the foot positioning is required. For example, 160 the user may want to respect different walking gaits depending ${ }_{161}$ on the terrain, to make the character step over stones to cross 162 a river, or walk through some space full of holes whilst avoid${ }_{163} \mathrm{ing}$ falling. For this purpose we have developed a framework to 164 animate virtual characters following footstep trajectories, while 165 still being able to follow trajectories based on the movement of ${ }_{166}$ the COM when necessary.

${ }_{167}$ Online locomotion systems [9] traditionally produce syn168 thesized motions that follow a COM trajectory, with procedural 169 corrections for uneven terrain. These methods can nicely fol170 low COM trajectories, but they lack control over the style of 171 walking and the kind of steps. For instance, we cannot control 172 whether in order to walk fast, the character will move with large ${ }_{173}$ distances between steps or with a fast sequence of short steps. 174 This is the main issue we address in our work: to provide an 175 animation system that is able to accurately follow footstep tra176 jectories while meeting real-time constraints, and that can scale 177 to handle large groups of animated characters .

${ }_{178}$ For this purpose, we introduce two parametric spaces based 179 on the position of each foot: $\Omega_{f_{L}}$ and $\Omega_{f_{R}}$, and switch between 180 the two depending on the swing foot, as well as a parametric ${ }_{181}$ space based on the root movement $\Omega_{f_{R}}$. Our technique takes 182 into account both displacement (from $\Omega_{f_{L}}$ and $\Omega_{f_{R}}$ ) and speed ${ }_{183}$ (from $\Omega_{r}$ ) to ensure the satisfaction of both spatial and temporal ${ }_{184}$ constraints. Our system provides the user with the flexibility 185 to choose between different control granularities ranging from ${ }_{186}$ exact foot positioning to exact root velocity trajectories. Fig. 2 187 shows our framework.

\section{4. Footstep-based Locomotion}

189 The main goal of the Footstep-based Locomotion Controller 190 is to accurately follow a footstep trajectory, i.e., to animate a 191 fully articulated virtual human to step over a series of foot192 plants with space and velocity constraints. The system must
193 meet real-time constraints for a group of characters, should be 194 robust enough to handle sparse motion clips, and needs to pro195 duce synthesized results that are void of artifacts such as foot 196 sliding and collisions.

\section{4.1. Motion Clip Analysis}

From a collection of cyclic motion clips ${ }^{1}$, we need to extract 199 individual footsteps. Each motion clip contains two steps, one 200 starting with the left foot on the floor, and one starting with 201 the right foot. A step is defined as the action where one foot 202 of the character starts to lift-off the ground, moves in the air 203 and finishes when it is again planted on the floor. We say that 204 a footstep corresponds to one foot when that foot is the one 205 performing the action previously described. The foot that stays 206 in contact with the floor for most of the duration of the footstep 207 is called the supporting foot, since it supports the weight of the 208 body. This applies even for running motions, where the support 209 foot goes into fly mode for a short phase of the footstep, but it is 210 still the one supporting the weight during most of the footstep.

During an offline analysis, each motion clip $m_{i}$ is annotated 212 with the following information: (1) $\mathbf{v}_{i}^{r}$ : Root velocity vector. (2) ${ }_{213} \mathbf{d}_{i}^{L}$ : Displacement vector of the left foot. (3) $\mathbf{d}_{i}^{R}$ : Displacement 214 vector of the right foot.

Similar to [9], animations are analyzed in place, that is, we 216 ignore the original root forward displacement, but keep the ver217 tical and lateral deviations of the motion. This allows an auto218 matic detection of foot events, such as lifting, landing or plant${ }_{219}$ ing, from which we can deduce the displacement vector of each 220 foot. For example, the displacement vector of the left foot $\mathbf{d}_{i}^{L}$ 221 is obtained by subtracting the right foot position at the instant 222 of time when the left foot lands, from the right foot position ${ }_{223}$ at the instant of time when the left foot is lifting off. These 224 displacements will be later used to move the whole character, ${ }_{225}$ eliminating any foot sliding. By adding $\mathbf{d}_{i}^{L}$ to $\mathbf{d}_{i}^{R}$ and knowing 226 the time duration of the clip, we can calculate the average root 227 velocity vector $\mathbf{v}_{i}^{r}$ of the clip $m_{i}$.

This average velocity is used to classify and identify an229 imations, by providing an example point which is the input 230 for the polar gradient band interpolator ( where each example ${ }_{231}$ point represents a velocity in a 2D parametric space). Gradi${ }_{232}$ ent band interpolation specifies an influence function associ233 ated with each example, which creates gradient bands between ${ }_{234}$ the example point and each of the other example points. These 235 influence functions are normalized to get the weight functions 236 associated with each example. However the standard gradient 237 band interpolation is not well suited for interpolation of exam${ }_{238}$ ples based on velocities. The polar gradient band interpolation 239 method is based on reasoning that in order to get more desir240 able behavior for the weight functions of example points that ${ }_{241}$ represent velocities, the space in which the interpolation takes 242 place should take on some of the properties of a polar coordi${ }_{243}$ nate system. It allows for dealing with differences in direction

\footnotetext{
${ }^{1}$ Although cyclic animations are not strictly required by our method, they help find smoother transitions between consecutive footsteps and are preferred by most standard animation systems [9]
} 


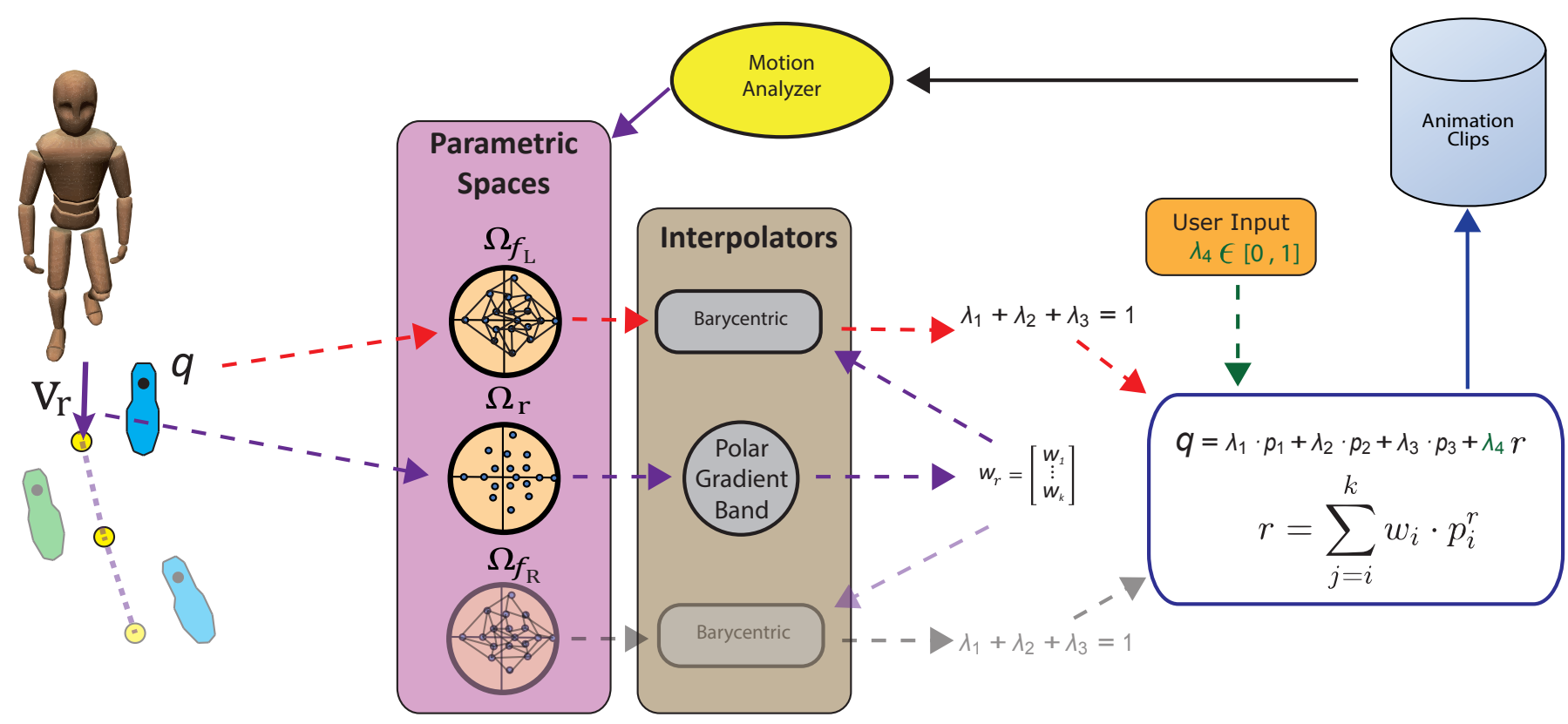

Figure 2: Online selection of the blend weights to accurately follow a footstep trajectory. $\Omega_{r}$ uses a gradient band polar based interpolator [9] to give a set of weights $w_{j}$, which are then used by the barycentric coordinates interpolator to tradeoff between footstep and COM accuracy.

244 and magnitude rather than differences in the Cartesian vector 245 coordinate components. For more details we refer the reader to 246 [9].

247 Each motion clip is then split into two animation steps $A_{i}^{L}$ ${ }_{248}$ for the left foot and $A_{i}^{R}$ for the right foot. For each foot, we need 249 to calculate all the possible positions that can be reached based 250 on the set of animation steps available. Since the same analysis ${ }_{251}$ is performed for both feet separately, from now on we will not 252 differentiate between left and right for the ease of exposition. ${ }_{253}$ For each individual step animation $A_{i}$ and given an initial root ${ }_{254}$ position, we want to extract the foot landing position $p_{i}$, if the 255 corresponding section of its original clip was played. This is 256 calculated by summing the root displacement during the sec257 tion of the animation with the distance vector between the root 258 projection over the floor and the foot position in the last frame. ${ }_{259}$ The set $\left\{p_{i} \mid \forall i \in[1, n]\right\}$ where $n$ is the number of step an260 imations, provides a point cloud. Fig. 3 shows the Delaunay ${ }_{261}$ triangulation that is calculated for the point cloud of landing 262 positions. This triangulation is queried in real time to deter263 mine the simplex that contains the next footstep in the input ${ }_{264}$ trajectory. Once the triangle is selected, we will use its three 265 vertices $p_{1}, p_{2}$ and $p_{3}$ to compute the blending weights for each ${ }_{266}$ of the corresponding animations $A_{1}, A_{2}$ and $A_{3}$.

\section{4.2. Footstep and Root Trajectories}

268 Our system can work with both footstep trajectories and ${ }_{269} \mathrm{COM}$ trajectories. A footstep trajectory will be given as an or270 dered list of space-time positions with orientations, whether it 271 is precomputed or generated on-the-fly.

${ }_{272}$ The input footstep trajectory may be accompanied by its as${ }_{273}$ sociated root trajectory (a space-time curve, rather than a list of 274 points, and an orientation curve), or else we can automatically 275 compute it from the input footsteps by interpolation. This is

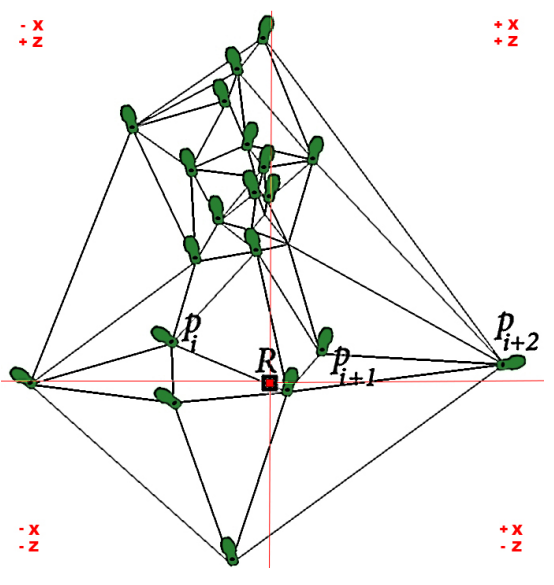

Figure 3: Delaunay triangulation for the vertices representing the landing positions $\left(p_{i}, p_{i+1}, p_{i+2}, \ldots\right)$ of the left foot when the root, $R$ is kept in place.

276 achieved by computing the projection of the root on the ground 277 plane, as the midpoint of the line segment joining two consec278 utive footsteps. The root orientation is then computed as the 279 average between the orientation vectors of each set of consec280 utive steps. This provides us with a sequence of root positions 281 and orientations which can be interpolated to approximate the 282 motion of the root over the course of the footstep trajectory.

\section{4.3. Online Selection}

During run time, the system animates the character towards 285 the current target footstep. If the target is reached, the next foot286 step along the trajectory is chosen as the next target. For each ${ }_{287}$ footstep $q_{j}$ in the input trajectory $\left\{q_{1}, q_{2}, q_{3}, \ldots, q_{m}\right\}$ we need to 288 align the Delaunay triangulation graph with the current root po289 sition and orientation. Then the triangle containing the next foot 
290 position is selected as the best match to calculate the weights re291 quired to nicely blend between the three animations in order to 292 achieve a footstep that will land as close as possible to the de${ }_{293}$ sired destination position $q_{j}$ (Fig. 4). Notice that these weights 294 are applied equally to all the joints in the skeleton, which means 295 that at this stage we cannot accurately adjust the specific foot 296 orientation required by each footstep in the input trajectory.

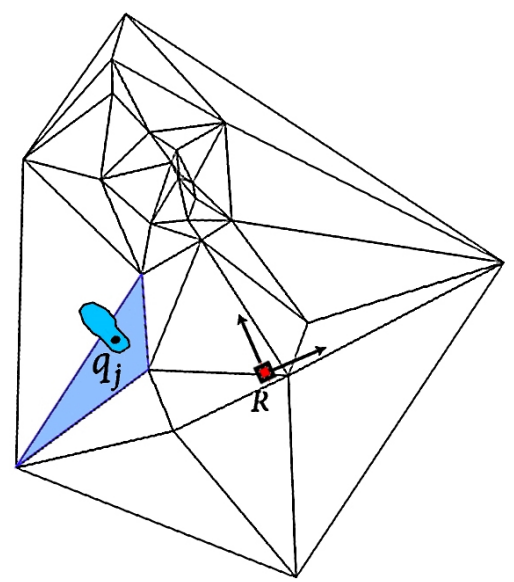

Figure 4: By matching root position and orientation, we can determine the triangle containing the destination position for the landing position $q_{j}$.

\section{4.4. Interpolation}

298 Footstep parameters change between successive footplants, 299 remaining constant during the course of a single footstep (sev300 eral frames of motion). Therefore we need to compute the best 301 interpolation for each footstep, blend smoothly between con302 secutive steps, and apply the right transformation to the root in 303 order to avoid foot-sliding or intersections with the ground.

${ }_{304}$ To meet these requirements, we use a barycentric coordi305 nates based interpolator in $\Omega_{f_{L}}$ and $\Omega_{f_{R}}$, and constrain the so306 lution based on the weights computed in $\Omega_{r}$. This allows us to 307 animate a character at the granularity of footsteps, while simul308 taneously accounting for the global motion of the full body.

309 If we only consider the footstep parametric space, then the 310 vertices of the selected triangle are those that can provide the 311 best match for the desired foot position. The barycentric co312 ordinates of the desired footstep are calculated for the selected ${ }_{313}$ triangle as the coordinates that satisfy:

$$
\begin{array}{r}
q_{j}=\lambda_{1} \cdot p_{1}+\lambda_{2} \cdot p_{2}+\lambda_{3} \cdot p_{3}, \\
\lambda_{1}+\lambda_{2}+\lambda_{3}=1
\end{array}
$$

${ }_{314}$ where $p_{1}, p_{2}$ and $p_{3}$ are the positions of the foot landing if we 315 run animation steps $A_{1}, A_{2}$ and $A_{3}$ respectively. The calculated ${ }_{316}$ barycentric coordinates are then used as weights for the blend317 ing between animations. A nice property of the barycentric co318 ordinates is that the sum equals 1 , which is a requirement for 319 our blending. Finally in order to move the character towards 320 the next position, we need to displace the root of the character 321 adequately to avoid foot sliding. The final root displacement

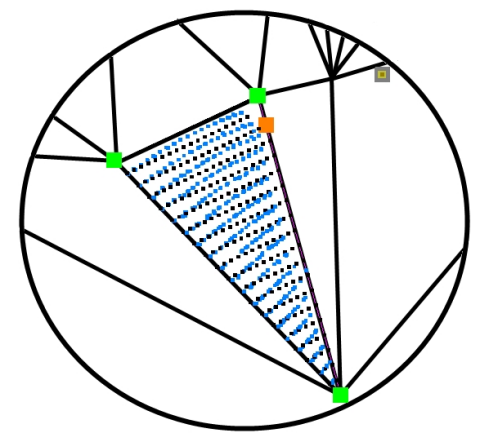

Figure 5: Offsets for different landing positions in a triangle, between barycentric coordinates interpolation (black dots) and blending the whole skeleton using SLERP (blue dots).

322 vector, $\mathbf{d}_{j}^{r}$ is calculated as the weighed sum of the root's dis323 placement of the three selected animation steps (Eq. 2), and ${ }_{324}$ changes in orientation of the input root trajectory are applied as 325 rotations over the ball of the supporting foot.

$$
\mathbf{d}_{j}^{r}=\lambda_{1} \cdot \mathbf{d}_{1}^{r}+\lambda_{2} \cdot \mathbf{d}_{2}^{r}+\lambda_{3} \cdot \mathbf{d}_{3}^{r}
$$

326

This provides a final root displacement that is the result of 327 interpolating between the three root displacements in order to 328 avoid any foot sliding. It is important to notice that the barycen329 tric coordinates provide the linear interpolation required be${ }_{330}$ tween three points in $2 \mathrm{D}$ space to obtain the position $q_{j}$. This ${ }_{331}$ is an approximation of the real landing position that our char332 acter will reach, as the result of blending the different poses of ${ }_{3 з 3}$ the three animation clips, using spherical linear interpolation ${ }_{334}$ (SLERP) with a simple iterative approach as described in [30].

Therefore there will be an offset between the desired posiз36 tion $q_{j}$ and the position reached after interpolating the three an${ }_{337}$ imations. To illustrate this offset, Fig. 5 shows the points sam${ }_{338}$ pled to compute barycentric coordinates in black, and in blue ззя the real landing positions achieved after applying the barycen340 tric weights to the animation engine and performing blending ${ }_{341}$ using SLERP. In order to correct this small offset at the same 342 time that we adjust the feet to the elevation of the terrain and ${ }_{343}$ orient the footstep correctly, we incorporate a fast and simple ${ }_{344}$ IK solver.

\section{4.5. Inverse Kinematics}

346 An analytical IK solver modifies the leg joints in order to ${ }_{347}$ reach the desired position at the right time with a pose as close 348 as possible to the original motion capture data. For footstep${ }_{349}$ based control, the desired foot position is already encoded in the 350 footstep trajectory, and for COM trajectories the final position is ${ }_{351}$ calculated by projecting the current position of the foot over the 352 terrain. The controller feeds the IK system with the end position 353 and orientation for each footstep. This allows the system to 354 handle footsteps on uneven terrain.

\section{5. Incorporating Root Movement Fidelity}

In some scenarios the user may be more interested in fol357 lowing root velocities than in placing the feet at exact footsteps 
358 or with specific walking styles. We present a solution to include 359 root movement based interpolation in our current barycentric 360 coordinates based interpolator through a user controlled param${ }_{361}$ eter $\lambda_{4}$.

362 For this purpose, we incorporate the locomotion system pre363 sented by Johansen [9] to produce synthesized motions that 364 follow a COM trajectory with correction for uneven terrain. ${ }_{365}$ During offline analysis, a parametric space is defined using all 366 the root velocity vectors extracted from the clips in the motion 367 database. For example, a walk forward clip at $1.5 \mathrm{~m} / \mathrm{s}$, and a 368 left step clip at $0.5 \mathrm{~m} / \mathrm{s}$ produces a parametric space using the 369 root velocity vectors going from the forward direction to the $37090^{\circ}$ direction, and with speeds from $0.5 \mathrm{~m} / \mathrm{s}$ to $1.5 \mathrm{~m} / \mathrm{s}$.

${ }_{371}$ Given a desired root velocity we define a parametric space ${ }_{372} \Omega_{r}$, and a gradient band interpolator in polar space [9] is created 373 to compute the weights for each animation clip to produce the 374 final blended result. The gradient band interpolator does not en375 sure accuracy of the produced parameter values but it does en376 sure smooth interpolation under dynamically and continuously 377 changing parameter values, as with a player-controlled char378 acter. Once the different clips are blended with the computed 379 weights, the system predicts the support foot position at the end 380 of the cycle and projects it on the ground to find the exact posi${ }_{381}$ tion where it should land.

382 The root movement based interpolator will select a set of $k$ ${ }_{383}$ animations $A_{1}^{r}$ to $A_{k}^{r}$ with their corresponding weights: $w_{1}, \ldots, w_{k}$. ${ }_{384}$ Each of those animations provides a landing position $p_{1}^{r}, \ldots, p_{k}^{r}$, 385 and if we only interpolated these animations we would obtain 386 the landing point $r$.

387 In order to incorporate the output of the polar gradient band 388 interpolator in the barycentric coordinates based interpolator 389 we proceed as indicated in Algorithm 1.

390 The algorithm first checks whether a vertex of the current 391 triangle $\left\langle p_{1}, p_{2}, p_{3}\right\rangle$ can be replaced by any of the three vertices ${ }_{392}$ with highest weights selected by the polar band interpolator, $p_{j}^{r}$, ${ }_{393} j \in[1, k]$ (lines $1-13$ in the algorithm). This replacement takes ${ }_{394}$ place if the distance between the two landing positions $p_{i}$ and ${ }_{395} p_{j}^{r}$ is within a user input threshold $\epsilon$ (line 7), and the resulting 396 triangle still contains the desired landing position $q_{j}$ (function ${ }_{397}$ IsInTriangle returns true if $q_{j}$ is inside the new triangle). This 398 means that there is another animation that also provides a valid 399 triangle and has a root velocity that is closer to the input root 400 velocity.

401 Next, function CalculateRootLanding computes the landing 402 position reached after blending the animations given by the root 403 movement interpolator (Eq. 3).

$$
r=\sum_{i=1}^{k} w_{i} \cdot p_{i}^{r}
$$

(3) ${ }_{423}$ Time Warping. Incorporating root velocity in the interpola-

404 Finally, ComputeWeights calculates the three $\lambda_{i}$ for the next 405 footstep $q_{j}$ by incorporating a user provided $\lambda_{4}$ and the result of 406 the polar band interpolator $r$ (Eq. 4).

$$
q_{j}=\lambda_{1} \cdot p_{1}+\lambda_{2} \cdot p_{2}+\lambda_{3} \cdot p_{3}+\lambda_{4} \cdot r
$$
${ }_{424}$ tion, does not always guarantee that the time constraints as-

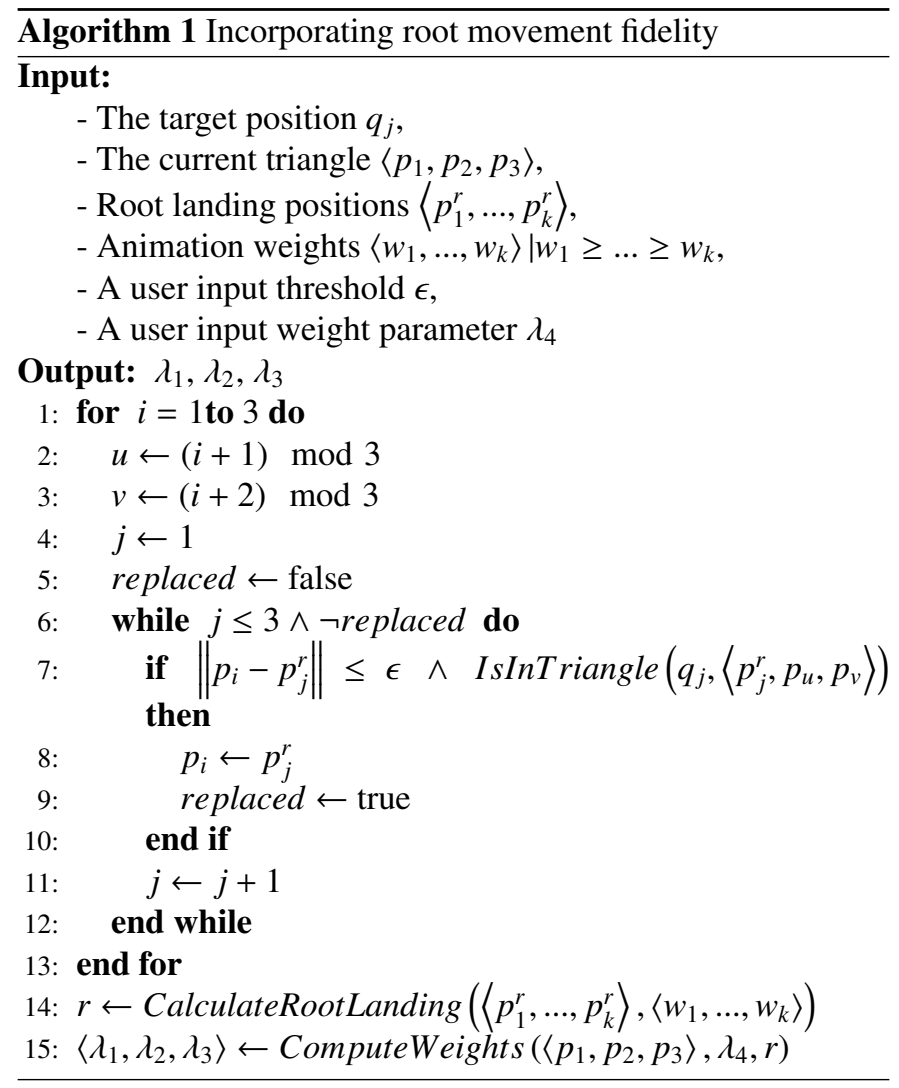

and $\lambda_{i}$ are defined using the following relationship:

$$
\lambda_{1}+\lambda_{2}+\lambda_{3}+\lambda_{4}=1
$$

407 Since $w_{i}$ and $p_{i}^{r}$ are known $\forall i \in\{1, \ldots, k\}$, and $\lambda_{4}$ is a user in408 put, we have a linear system, where $\lambda_{4}$ determines the trade-off 409 between following footsteps accurately (if $\lambda_{4}=0$ ), and simply ${ }_{410}$ following root movement (if $\lambda_{4}=1$ ).

As the user increases $\lambda_{4}$ there will be a value $\beta \in[0,1]$ for 412 which $\lambda_{1}, \lambda_{2}$ or $\lambda_{3}$ will be negative, when solving the system 413 of equations formed by eq. 4 and eq.5. In order to avoid anima414 tion artifacts it is necessary to deal only with positive weights, ${ }_{415}$ therefore we guarantee that the system will only reproduce $q_{j}$ ${ }_{416}$ accurately as long as $\lambda_{4}<\beta$. If we further increase $\lambda_{4}$ beyond ${ }_{417}$ the value $\beta$ then the algorithm will provide the blending values 418 that correspond to a new point $q^{\prime}$ which is the result of a linear ${ }_{419}$ interpolation between $q_{j}$ and point $r$. When $\lambda_{4}=1$ the result420 ing blending will be exclusively the one provided by the root ${ }_{421}$ movement trajectory since $\lambda_{1}=\lambda_{2}=\lambda_{3}=0$. Fig. 6 illustrates 422 this situation.

425 signed per footstep will be satisfied. Therefore once we have 426 the final set of animations to interpolate between, with their ${ }_{427}$ corresponding weights $\lambda_{i}, i \in\{1,2,3\}$ and $w_{j}, j \in[1, k]$, we 428 need to apply time warping. Each input footstep $f_{m}$ has a time ${ }_{429}$ stamp $\tau_{m}$ indicating the time at which position $q_{m}$ should be 430 reached (where $m \in[1, M]$ and $M$ is the number of footsteps in ${ }_{431}$ the input trajectory). The total time of the current motion, $T$ can 


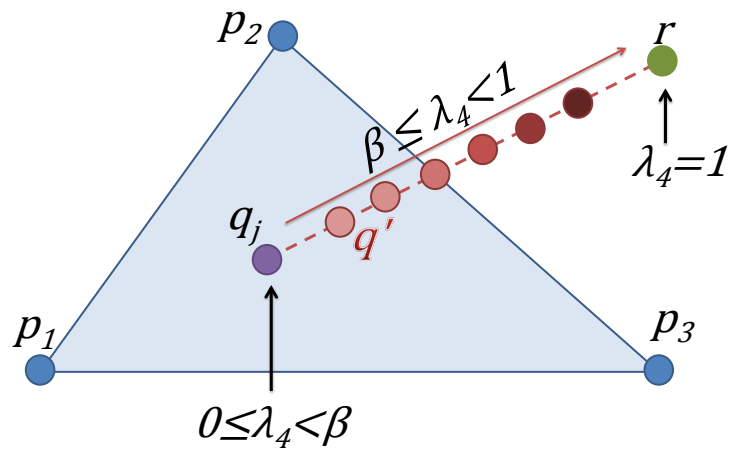

Figure 6: When solving the system of equations given by eq.4 and eq.5, the value of either $\lambda_{1}, \lambda_{2}$ or $\lambda_{3}$ will be negative when $\lambda_{4} \geq \beta$. Therefore we need to calculate the barycentric coordinates for a new point $q^{\prime}$ which moves linearly from $q_{j}$ to $r$ as the user increases the value of $\lambda_{4}$ from $\beta$ to 1 . This means solving the system of equations for $q^{\prime}$ instead of $q_{j}$, as it is the closest point to the desired landing position which guarantees that all weights in eq. 5 will be positive.

432 be calculated as the weighted sum of the time of the animation ${ }_{433}$ steps being interpolated: $T=\sum_{i=1}^{3}\left(\lambda_{i} \cdot t\left(A_{i}\right)\right)+\sum_{j=1}^{k}\left(w_{j} \cdot t\left(A_{j}\right)\right)$. ${ }_{434}$ Therefore the time warping factor that needs to be applied can 435 be calculated as: $\operatorname{warp}_{m}=\left(\tau_{m}-\tau_{m-1}\right) / T$.

${ }_{436}$ Outside the Convex-Hull. The footstep parametric space de${ }_{437}$ fines a convex-hull delimiting the area where our character can 438 land its feet. When our target footstep position falls inside this 439 area, clips can be interpolated to reach that desired position. ${ }_{440}$ But if it falls outside this convex-hull we still want the system to ${ }_{441}$ consider and try to reach it. Our solution to handle this problem 442 consists of projecting orthogonally the input landing position $q$ 443 over the convex-hull to a new position $q_{p r o j}$. Our system then ${ }_{444}$ gives the blending weights for $q_{\text {proj }}$ and applies IK to adjust the 445 final position. We include a parameter to define a maximum ${ }_{446}$ distance for the IK to set an upper limit on the correction of the ${ }_{447}$ landing position. It is important to notice that even if the in448 put trajectory has some footsteps that are unreachable with the ${ }_{449}$ current data base of animation clips, our system will provide 450 a synthesized animation that will follow the input trajectory as ${ }_{451}$ closely as possible, until it recovers and catches up with future ${ }_{452}$ steps in the input trajectory. This situation is similar to the sce${ }_{453}$ narios where the user increases $\lambda_{4}$ and then reduces it again.

\section{${ }_{454}$ 6. Results}

455 The animation system described in the paper is implemented 456 in C\# using the Unity 3D Engine [31]. The footstep trajectories 457 used to animate the characters are generated using the method 458 described in [7] or are created by the user. Some difficult sce459 narios, exercising careful footstep selection, are shown in Fig. 4601 and Fig. 7. Agents carefully plant their feet over pillars (Fig. 461 7-a) or use stepping stones to avoid falling into the water (Fig. 462 7-b). We show our ability to handle over a hundred agents at 13 ${ }_{463}$ FPS (Fig. 7-c and Fig. 9). The supplementary video demon-
${ }_{464}$ strates additional results ( high resolution video ${ }^{2}$, low resolution 465 video $^{3}$ ).

${ }_{466}$ Obstacle Course. We exercise the locomotion dexterity of a ${ }_{467}$ single animated character in an obstacle course. The character 468 follows a footstep trajectory with different walking gaits , alter469 nating running and walking phases (Fig. 1-a,b), and including 470 sidesteps (Fig. 1-c) and backward motion (Fig. 1-e).

${ }_{471}$ Stepping Stone Problem. Stepping stone problems (Fig. 7472 b) require careful footstep level precision where constraints re473 quire the character to place their feet exactly on top of the stones 474 in order to successfully navigate the environment. Our frame475 work can be coupled with footstep-based controllers to solve 476 these challenging benchmarks.

477 Integration with Crowd Simulator. We integrate our ani478 mation system with footstep-based simulators [7]; our charac479 ter follows the simulated trajectories without compromising its 480 motion fidelity while scaling to handle large crowds of charac481 ters (Fig. 7-c).

482 It is important to mention that the quality of the results de483 pends strongly on the quality of the clips available from the 484 motion capture library. As can be seen in the video, the least 485 precise movements in our results are side steps and back steps. ${ }_{486}$ This is due to two reasons: (1) we had a small number of an487 imations compared to other walking gaits, and thus triangles 488 covering that space have larger areas, and (2) interpolation ar489 tifacts appear when blending between animations that move in 490 opposite directions (for example a backwards step with a for491 ward step). We believe that having a better and denser sam492 pling in these areas will improve the results. For steps falling in 493 triangles of smaller areas, and with all the vertices in the same 494 quartile we have obtained results of high quality even for diffi495 cult animations such as running or performing small jumps.

\section{6.1. Foot Placement Accuracy}

497 The presented barycentric coordinates interpolator assumes 498 a small offset between the results of linearly interpolating land499 ing positions from the set of animations being blended, and the 500 actual landing position when calculating spherical linear inter${ }_{501}$ polation over the set of quaternions. This small offset depends 502 on the area of the triangle, so as we incorporate more anima503 tions into our data base, we obtain a denser sampling of landing ${ }_{504}$ positions and thus reduce both the area of the triangles and the 505 offset. We believe this is a convenient trade off since such a 506 small offset can be eliminated with a simple analytical solver 507 but the efficiency of computing barycentric coordinates offers 508 great performance. It is also important to notice that if exact 509 foot location is not necessary, and the user only needs to indi510 cate small areas for stepping as in the water scenario, then it is 511 not necessary to apply the IK correction. Fig. 8 shows the offset 512 between the landing position and the footstep. The magnitude 513 of the error is illustrated as the height of the red cylinders that 514 are located at the exact location where the foot first strikes.

\footnotetext{
${ }^{2}$ https://www.dropbox.com/s/o1b9w73qd45fmip/videoCAG.mp4?dl=0

${ }^{3}$ https://www.dropbox.com/s/ptdz788f2k9ad3g/videoCAGlowRes.mp4?dl=0
} 


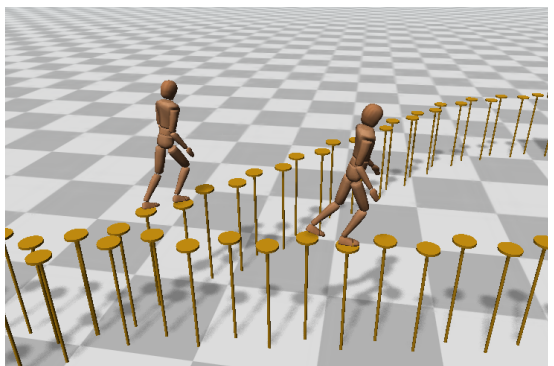

(a)

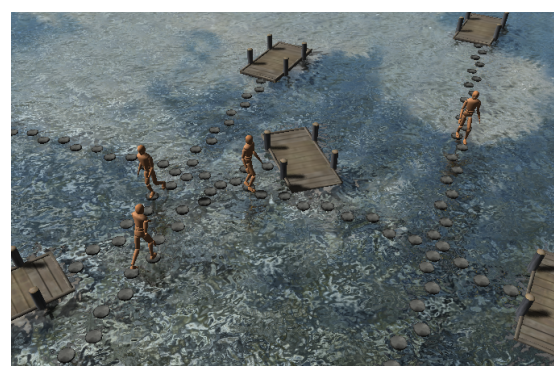

(b)

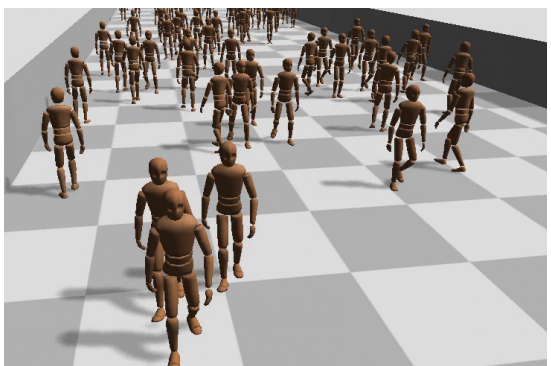

(c)

Figure 7: (a) Agents accurately following a footstep trajectory and avoiding falls by carefully stepping over pillars. (b) The stepping stone problem is solved with characters avoiding falls into the water. (c) A crowd of over 100 agents simulated at interactive rates.

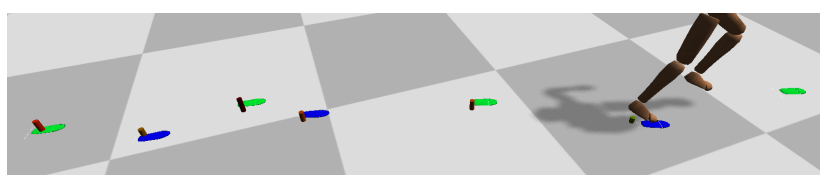

Figure 8: The red columns show the small offset between landing position and the footstep when the IK corrections are not being applied.

\section{6.2. Performance}

516

Fig. 9 shows the frame rate we obtain as we double the 517 number of agents. It is important to notice that increasing the 518 number of animations would enhance the quality and accuracy 519 of the results, with just a small overhead on the performance.

${ }_{520}$ The average time of the locomotion controller is $0.43 \mathrm{~ms}$, ${ }_{521}$ this process includes blending animations, IK, the polar band 522 interpolator and our barycentric coordinates based interpolator. ${ }_{523}$ The computational cost of our footstep interpolator is $0.2 \mathrm{~ms}$, 524 which is amortized over several frames as the interpolation in ${ }_{525} \Omega_{f_{L}}$ or $\Omega_{f_{R}}$ only need to be performed once per footstep. This 526 time is divided between computing the root movement polar 527 band interpolator which takes $0.155 \mathrm{~ms}$ and our barycentric co528 ordinates interpolator which takes $0.045 \mathrm{~ms}$. Performance re529 sults were measured on an Intel Core i7-2600k CPU at $3.40 \mathrm{GHz}$ 530 with 16 GB RAM.

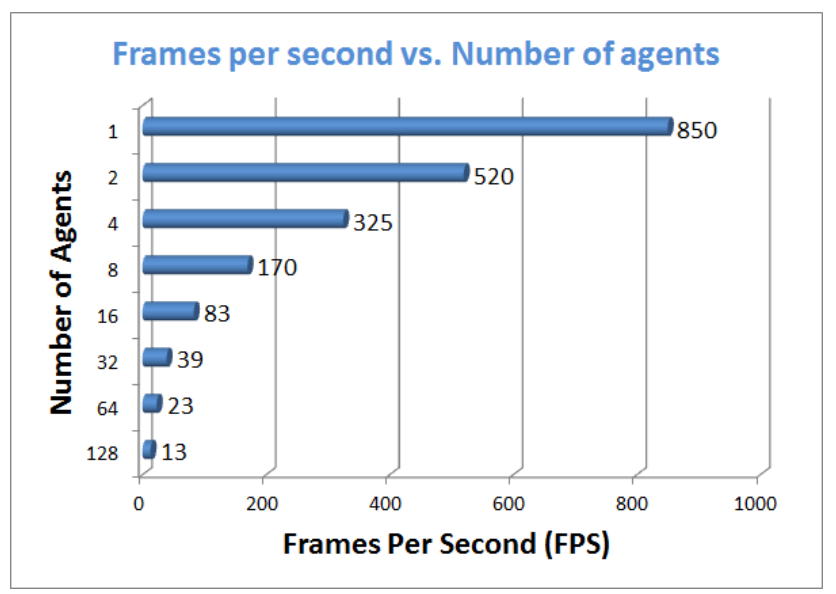

Figure 9: Performance of the Footstep Locomotion System in frames per second as the number of agents increases.

\section{${ }_{531}$ 7. Conclusions and Future Work}

532 We have presented a system that uses multiple parameter 533 spaces to animate fully embodied virtual humans to accurately 534 follow a footstep trajectory respecting root velocities, using a 535 relatively small number of animation clips (24 in our exam${ }_{536}$ ples). Our method is fast enough to be used with tens of char${ }_{537}$ acters in real time (25 FPS) and over a hundred characters at 53813 FPS. The method can handle uneven terrain, and can be eas539 ily extended to introduce additional locomotion behaviors by 540 grouping new sets of animation clips and generating different ${ }_{541}$ parametric spaces. For example, walking and running motions 542 can be blended together, but if we wanted to add crawling mo543 tions or jumping motions, it would be better to separate them in 544 different parametric spaces for each style. This will avoid un545 natural interpolations that can appear when blending between 546 very different styles. Having different parametric spaces re${ }_{547}$ quires some sort of classification, which could initially be done 548 manually but it could also be based on the characteristics of the 549 motion, such as changes in acceleration, maximum heights of 550 the root, length of fly phase, etc. Assuming we can extract the ${ }_{551}$ parametric spaces for different animation types, it would also 552 be necessary in some cases to have additional transition clips to 553 switch between very different locomotion types, i.e. crawling 554 and walking.

555 We do not run physical or biomechanical simulations, and 556 use interpolation and blending between motion capture anima557 tions. Our method accuracy depends on the variety of animation 558 clips, while its quality and efficiency depends on the number of 559 clips. A trade-off between efficiency and accuracy is therefore 560 necessary, for which we have found a good equilibrium.

${ }_{561}$ Limitations. In order to reduce the dimensionality of the prob562 lem, we have not included in our parametric space the orienta563 tion of the previous footstep. Ignoring the final orientation of 564 the character at the end of the previous step can induce some 565 discontinuities between footsteps. We mitigate this effect by 566 blending between footsteps automatically for a small amount of 567 time (about 0.2 seconds) at the advantage of reducing the com568 putational time and thus making our method suitable for large 569 groups of agents in real time. Regarding the selection of ani570 mation at the end of each footstep, notice that in our database, 571 left and right animation steps are extracted from complete ani572 mation cycles that are usually consistent in parameters such as 
573 velocity, acceleration and walking gait. Therefore for a given 574 sequence of steps, the most likely animation steps to be chosen 575 will be those extracted from the same set of animation cycles, 576 thus resulting in smooth and natural transitions between very 577 similar steps. When the characteristics of the steps change dras578 tically, then our method needs to blend between steps from very 579 different animation cycles. So in general, alternating left/right 580 steps results in natural transitions with smooth continuity when ${ }_{581}$ blending animations, and only when the input step trajectory 582 changes drastically between each pair of steps, we may observe 583 transitions between animations that feel unnatural. This can 584 happen if the step trajectory is done manually with artifacts due 585 to the user's lack of experience creating footstep trajectories, 586 or for example when the input trajectory forces the character to ${ }_{587}$ walk over artificially located steps, like crossing a river by step${ }_{588}$ ping over stones. We would like to emphasize that this situation 589 would also look awkward in the real world and thus the result 590 of our synthesized animation may be the desired one.

${ }_{591}$ Future Work. For future work we would like to extend our 592 barycentric coordinates interpolator to $3 \mathrm{D}$ space with the third 593 coordinate being the root velocity. This will free our system 594 from the polar band interpolator which not only takes longer to 595 compute but also selects too many animations which results in ${ }_{596}$ slower blending. One thing to explore could be to interleave the 597 execution of the Footstep-based Locomotion Controller from 598 different characters in different frames, ensuring we do not ex599 ecute it for all the agents in the crowd.

\section{Acknowledgements}

601 This work has been partially funded by the Spanish Ministry 602 of Science and Innovation under Grant TIN2010-20590-C02603 01. A. Beacco is also supported by the grant FPUAP2009-2195 604 (Spanish Ministry of Education). The research reported in this $605 \mathrm{document} /$ presentation was also performed in connection with ${ }_{606}$ Contract Number W911NF-10-2-0016 with the U.S. Army Re607 search Laboratory. The views and conclusions contained in this 608 document are those of the authors and should not be interpreted 609 as presenting the official policies or position, either expressed 610 or implied, of the U.S. Army Research Laboratory, or the U.S. ${ }_{611}$ Government. Citation of manufacturers or trade names does not 612 constitute an official endorsement or approval of the use thereof. ${ }_{613}$ The U.S. Government is authorized to reproduce and distribute 614 reprints for Government purposes notwithstanding any copy615 right notation hereon.

\section{${ }_{616}$ References}

627

638 [10] L. Kovar, M. Gleicher, F. Pighin, Motion graphs, in: ACM SIGGRAPH, 2002, pp. 473-482.

640 [11] L. Zhao, A. Safonova, Achieving Good Connectivity in Motion Graphs, Graphical Models 71 (4) (2009) 139-152.

642 [12] C. Ren, L. Zhao, A. Safonova, Human Motion Synthesis with 643 Optimization-Based Graphs, Computer Graphics Forum 29 (2).

644 [13] J. Min, J. Chai, Motion Graphs++, ACM Transactions On Graphics 31 (6) (2012) 1.

6 [14] M. Lau, J. J. Kuffner, Precomputed search trees: planning for interactive goal-driven animation, in: ACM Symposium on Computer Animation, 2006, pp. 299-308.

[15] A. Witkin, Z. Popovic, Motion warping, in: ACM SIGGRAPH, 1995, pp. 105-108.

651 [16] S. Chenney, Flow tiles, in: ACM Symposium on Computer Animation, 2004, pp. 233-242.

653 [17] A. Sud, E. Andersen, S. Curtis, M. Lin, D. Manocha, Real-time path planning for virtual agents in dynamic environments, in: IEEE Virtual Reality, 2007, pp. 91-98.

[18] P. Glardon, R. Boulic, D. Thalmann, Robust on-line adaptive footplant detection and enforcement for locomotion, The Visual Computer 22 (3) (2006) 194-209.

9 [19] J. Pettré, J.-P. Laumond, T. Siméon, A 2-stages locomotion planner for digital actors, in: Proceedings of the 2003 ACM SIGGRAPH/Eurographics Symposium on Computer Animation, SCA '03, Eurographics Association, Aire-la-Ville, Switzerland, Switzerland, 2003, pp. 258-264.

[20] J. Pettre, J.-P. Laumond, A motion capture-based control-space approach for walking mannequins: Research articles, Computer Animation and Virtual Worlds 17 (2) (2006) 109-126.

7 [21] M. Felis, K. Mombaur, Using Optimal Control Methods to Generate Human Walking Motions, Motion in Games (2012) 197-207.

[22] Autodesk, 3d studio max,

www.autodesk.com/products/autodesk-3ds-max/overview (2014).

[23] M. Girard, A. A. Maciejewski, Computational modeling for the computer animation of legged figures, in: SIGGRAPH, ACM, 1985, pp. 263-270.

[24] J. Chai, J. K. Hodgins, Constraint-Based Motion Optimization Using A Statistical Dynamic Model, ACM SIGGRAPH.

675 [25] M. G. Choi, J. Lee, S. Y. Shin, Planning biped locomotion using motion capture data and probabilistic roadmaps, ACM Transactions on Graphics 22 (2) (2003) 182-203.

[26] H. Ko, N. I. Badler, Animating human locomotion with inverse dynamics, IEEE Computer Graphics \& Applications 16 (2) (1996) 50-59.

80 [27] M. van de Panne, From Footprints to Animation, Computer Graphics Forum 16 (4) (1997) 211-223.

682 [28] B. van Basten, P. W. A. M. Peeters, A. Egges, The Step Space : Example683 Based Footprint-Driven Motion Synthesis, Computer Animation and Vir684 tual Worlds 21 (May) (2010) 433-441.

685 [29] B. van Basten, S. Stüvel, A. Egges, A Hybrid Interpolation Scheme for 686 Footprint-Driven Walking Synthesis, Graphics Interface (2011) 9-16.

687 [30] A. Shoulson, N. Marshak, M. Kapadia, N. I. Badler, ADAPT : The Agent Development and Prototyping Testbed, ACM SIGGRAPH I3D.

689 [31] Unity, Unity - game engine (2014).

URL http://unity3d.com/ 